\title{
EFFETS VENTILATOIRES DE DEBITS CROISSANTS D'ALFATESINE CHEZ LE CHIEN
}

\author{
Jean-Henri Gaudy*, Claude Dauthier, Jean-François Boitier, et Françols Ferracci
}

\section{RÉSUMÉ}

Les auteurs étudient chez 10 chiens les effets de débits croissants d'alfatésine sur la

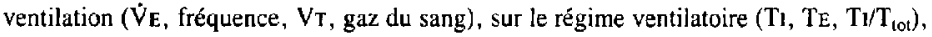
sur la neurogénèse ventilatoire ( $V_{T} / \mathrm{T}_{\mathrm{I}}$, pression d'occlusion à 0.5 secondes) et sur le réflexe de Breuer-Hering (durée de l'apnée après occlusion des voies aériennes en fin d'inspiration). Les résultats ont été comparés aux valeurs normales relevées dans la littérature. Une corrélation a été recherchée entre le débit d'alfatésine et les différents paramètres mesurés ou calculés. Pour de faibles débits d'alfatésine la ventilation est stimulée, stimulation traduite essentiellement par une tachypnée sans modification de VT. L'approfondissement de l'anesthésie s'accompagne d'une dépression respiratoire progressivement croissante (diminution de $\dot{V} \mathrm{E}$, de la fréquence, de VT/TI, de $\mathrm{P} 0.5$, augmentation de la $\mathrm{Paco}_{2}$ et de la durée d'apnée). Les mécanismes de la stimulation ventilatoire initiale et de la dépression respiratoire sont discutés. Les auteurs concluent que l'action centrale de l'alfatésine cst une stimulation du systeme nerveux central en cas d'anesthésie légère, puis une dépression croissante en fonction de la profondeur de l'ancsthésie.

Mots Clefs: ANesthésie, intraveineuse, alfatésine; CoMPliCATIONS, alfatésine, dépression respiratoire.

LES EFFETS ventilatoires des agents anesthésiques administrés par inhalation ou par voie intra-veineuse ont été très étudiés; les signes respiratoires constituent même un élément fondamental pour apprécier la profondeur de l'anesthésie dans le cas de la ventilation spontanée. .,2 $^{1}$

Les effets ventilatoires de l'alfatésine ont essentiellement été étudiés lors de l'injection de doses uniques ou répétées, ${ }^{3,9}$ alors que les effets de la perfusion continue de cet agent ont fait l'objet de très peu de travaux. La pharmacocinétique de l'alfatésine permet d'obtenir, par perfusion, des concentrations plasmatiques stables ${ }^{10}$ provoquant des anesthésies plus ou moins profondes. Le présent travail se propose d'étudier chez le chien les effets de la perfusion de débits croissants d'alfatésine sur la ventilation, sur le régime ventilatoire, sur la neurogénèse ventilatoire, et de rechercher une corrélation possible entre le débit d'alfatésine et les variations ventilatoires observées.

*J.H. Gaudy, Laboratoire d'anesthésiologie, Hôpital Rothschild 33, bd de Picpus 75012 PARIS. Département d'anesthésie Hôpital Saint-Antoine (Pr. C. Guilmet).

\section{Matériel et Methodes (Figure 1)}

L'étude a été réalisée chez 10 chiens, mâles, de race beagle (poids $16 \pm 3 \mathrm{~kg}$ ) à jeûn depuis 12 heures et non prémédiqués. Une veine du membre antérieur a été canulée (calibre 21) et l'anesthésie a été induite puis conduite avec de l'alfatésine diluée dans une solution isotonique de chlorure de sodium, administrée à l'aide d'une pompe êlectrique (Rhône Poulenc RPO4). Après induction de l'anesthésie, un tube trachéal à ballonnet (Portex $\mathrm{N}^{\circ} 8.5$ ) a été mis en place et la ballonnet a été gonflé de manière à assurer une étanchéité parfaite. Les animaux respirant spontanément de l'air ambiant ont été placés sur un matelas chauffant thermostaté, le température rectale étant maintenue à $37^{\circ} \mathrm{C}$. Une autre veine a été cathétérisée afin d'administrer éventuellement un soluté de remplissage vasculaire (Ringer Lactate). Un cathéter a été introduit par voie per cutanée dans une artère fémorale afin d'enregistrer la pression artérielle (capteur Statham P23 IA) et de recueillir des échantillons de sang pour la mesure de $\mathrm{PaO}_{2}$, de $\mathrm{Paco}_{2}$ et de pHa; les mesures ont été effectuées dans les cinq minutes qui suivaient le prélèvement (Instrument Laboratories Modele 313). La concentration fraction- 
GAUDY, et al.: EFFÉTS VENTILATOIRES DE DEBITS CROISSANTS D'ALFATÉSINE

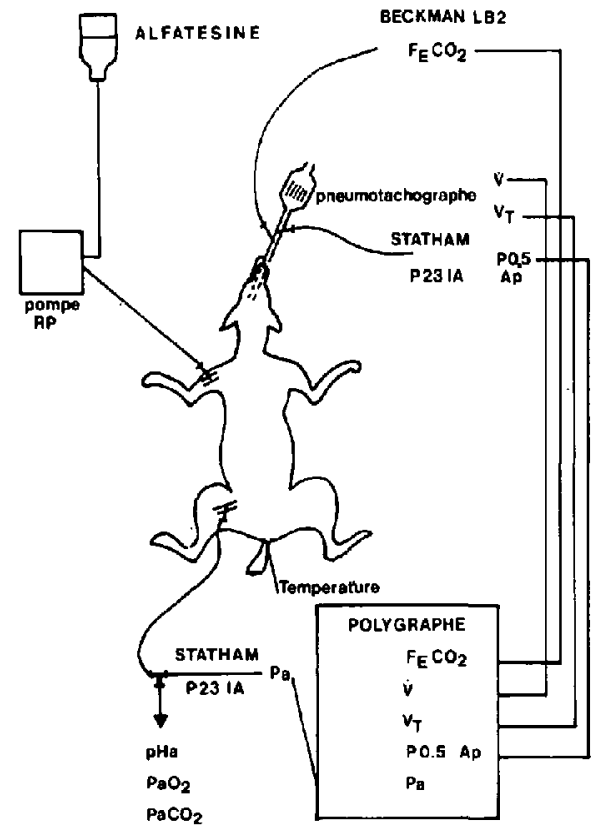

FIGURE 1 Représentation schématique des méthodes utilisées.

naire de gaz carbonique dans les gaz expirés $\left(\mathrm{FECO}_{2}\right)$ a été surveillée en continu (analyseur Beckman LB2). Le spirogramme a été obtenu après intégration du signal peumotachographique (Fleisch $\mathrm{N}^{\circ} 2$ ), ainsi que la pression dans les voies aériennes au niveau du tube trachéal (Capteur Statham P23 IA). La pression artérielle, $\mathrm{FECO}_{2}$, le spirogramme et la pression trachéale ont été enregistrés sur un polygraphe (Dynograph Beckman R 411) à la vitesse de $25 \mathrm{~mm} / \mathrm{sec}$.

\section{Mesures EfFectuées}

Après l'induction de l'anesthésie, le débit d'alfatésine a été ajusté de manière à obtenir une anesthésie légère, le niveau de l'anesthésie étant apprécié cliniquement. Chaque expérience a débuté après 60 minutes d'état stable jugé sur $\mathrm{FECO}_{2}$, la pression artérielle, la fréquence cardiaque, la fréquence respiratoire, le volume courant et la température. Puis le débit d'alfatésine a été augmenté par paliers successifs. Pour chaque palier, après 30 minutes d'état stable, les mesures suivantes ont été effectuées: gaz du sang artériel ( $\mathrm{pHa}, \mathrm{Paco}_{2}, \mathrm{PaO}_{2}$ ), volume courant (VT), durée de l'inspiration (TI), durée de l'expiration (TE), pression d'occlusion des voies aériennes en fin d'expiration, mesurée 0.5 secondes après le début de l'inspiration ( $\mathrm{P} 0.5$ ), durée de l'apnée après occlusion des voies aériennes en fin d'inspiration (Figure 2). Pour VT, Tı, TE, on a effectué la moyenne des mesures portant sur 10 cycles respiratoires consécutifs; pour P 0.5 , la moyenne de trois mesures séparées par au moins trois cycles respiratoires sans occlusion. $\mathrm{La}$ durée de l'apnée après occlusion des voies aériennes en fin d'inspiration a été corrigée et calculée pour un volume égal à $100 \mathrm{ml}$ (Figure 2). Le rapport du volume courant sur la durée de l'inspiration $(\mathrm{VT} / \mathrm{T} 1)$ et la ventilation minute $\left(V_{E}=V_{T} \times f\right)$ ont été calculés. Une corrélation a été recherchée entre le débit d'alfatésine et les différents paramètres mesurés ou calculés.

Chaque expérience a été interrompue soit en raison d'une hypoxie et d'une hypercapnie sévères, soit parce que le spirogramme devenait difficile à interpréter et à mesurer en raison d'un régime respiratoire très particulier (Figure 3), soit pour les deux raisons lorsque le niveau d'anesthésie devenait très profond.

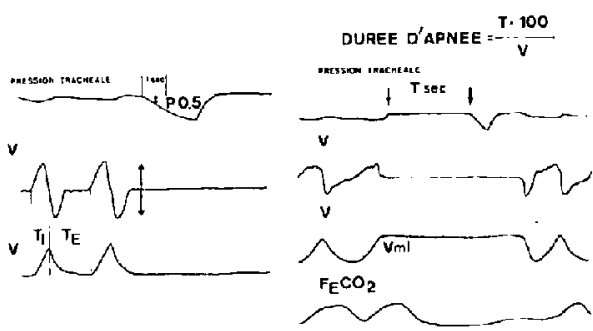

Figure 2 Mesure de la pression d'occlusion des voies aériennes au bout de 0.5 secondes (P 0.5 ), du volume courant, de la durée de l'inspiration Ti. de la durée de l'expiration (TE) et de la durée d'apnée après occlusion des voies aériennes en cours ou en fin d'inspiration.

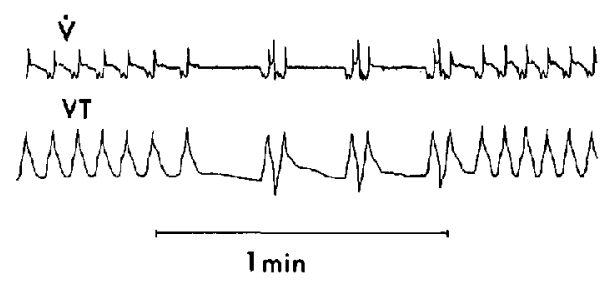

FigURE 3 Illustration du régime ventilatoire particulier observé chez un animal en fin d'expérience. $L$ 'inspiration se fait vers le haut. 


\section{RESULTATS}

L'induction de l'anesthésie s'accompagne constamment d'un rash cutané généralisé, d'un oedème du museau, des oreilles et des pattes, et d'une chute modérée de la pression artérielle (la pression artérielle systolique ne s'abaissant pas au-dessous de $9.31 \mathrm{kPa}$ ). Cette hypotension artérielle est facilement corrigée par l'administration d'un soluté de remplissage vasculaire (en moyenne 250 millilitres de Ringer Lactate). Ensuite la pression artérielle reste stable pour les plans superficiels de l'anesthésie. Puis quand l'anesthésie est approfondie, parallèlement à une hypercapnie progressivement croissante, la pression artérielle augmente, accompagnée d'une tachycardie. Le rash cutané disparait dans l'heure suivant l'induction, l'œdème persiste pendant toute la durée de l'expérience. Les débits d'alfatésine nécessaires pour obtenir une anesthésie superficielle (avec conservation des réflexes oculopalpébral, oculo-moteur, de déglutition, et quelquefois des mouvements des pattes) sont très variables d'un animal à l'autre (Tableau I). Il en est de même des débits au moment de l'arrêt de l'expérience. Les mêmes constatations s'appliquent aux valeurs des gaz du sang (Tableau I). En ce qui concerne le $\mathrm{pH}$ du sang artériel, il existe après l'induction une acidose métabolique ( $\mathrm{pHa}=7.28 \pm 0.04 ; \mathrm{Paco}_{2}$ $=4.6 \pm 0.57 \mathrm{kPa}$; valeurs normales chez le chien: $\left.\mathrm{pHa}=7.36 ; \mathrm{Paco}_{2}=5.05 \mathrm{kPa}\right) .{ }^{11}$ Cette acidose métabolique se complique d'une acidose respiratoire au fur et à mesure que l'on approfondit l'anesthésie.

EFfets sur la Ventilation (Vt, Ve, f, $\mathrm{Paco}_{2}$ ) (Figure 4).

Si on compare les résultats aux valeurs normales relevées par Green ${ }^{11}$ et Stahl ${ }^{12}$ chez des chiens de même poids, on constate qu'en anesthésie superficielle (débit d'alfatésine $<10 \mu \mathrm{l} \cdot \mathrm{kg}^{-1}$. min $^{-1}$ ) VE est augmenté ou normal. Le volume courant est le plus souvent normal, quelquefois augmente. $\mathrm{La} \mathrm{Paco}_{2}$ est soit normale, soit diminuée. Surtout, la fréquence respiratoire est augmentée.

Quand on approfondit l'anesthésie, la fréquence respiratoire diminue, VT ne varie pas, $\checkmark E$ diminue et la $\mathrm{PaCO}_{2}$ augmente. Quand on étudie la corrélation entre la débit d'alfatésine et ces paramètres, on constate (Tableaux II et III) que la diminution de VE est corrélée significativement avec l'augmentation du débit d'alfa- tésine $(\mathrm{r}=-0.471 \mathrm{p}<0.01)$ pour l'ensemble des animaux et individuellement pour neuf animaux sur 10. Pour VT il n'y a pas de corrélation pour l'ensemble des animaux, les valeurs individuelles allant dans le sens d'une corrélation soit positive, soit négative, soit qu'il n'y ait pas de corrélation significative. Pour la fréquence respiratoire, sa diminution est corrélée significativement avec le débit d'alfatésine pour l'ensemble des animaux ( $\mathrm{r}=-0.550 \mathrm{p}<$ 0.01 ) et individuellement pour sept animaux. L'augmentation de $\mathrm{Paco}_{2}$ est corrélée significativement avec l'augmentation du débit d'alfatésine pour l'ensemble des animaux $(r=0.490 p$ 0.01 ) et pour huit animaux sur dix.

EFFETS SUR LE REGIME VENTILATOIRE, SUR la Neurogénese Ventilatoire et SUR le Reflexe de BReuer Hering (Figures 5 et 6 ).

Les résultats observés n'ont pu être comparés à des données de la littérature concernant le chien éveillé, données qui n'existent pas à notre connaissance. Au fur et à mesure que le débit d'alfatésine augmente la fréquence respiratoire diminue sans variation significative du volume courant. La diminution de la fréquence est liée à l'augmentation de $\mathrm{TI}$ et à celle de $\mathrm{TE}, \mathrm{TE}$ augmentant plus que $T$, de sorte que le rapport $\mathrm{TI} / \mathrm{T}_{\text {tot }}$ diminue significativement. Les animaux ont présenté à un moment donné un régime respiratoire particulier (Figure 3). Pour quatre animaux il est survenu alors que le débit d'alfatésine était faible et que l'hypoxie et l'hypercapnie étaient modérées. Pour les autres animaux ce régime est apparu aux forts débits d'alfatésine alors qu'existaient une hypoxémie et une hypercapnic marquées. Ce régime est caractérisé par la succession plus ou moins régulière des évènements suivants: phase de respiration lente, régulière, avec un volume courant augmenté, pause respiratoire en expiration, suivie de deux ou trois mouvements respiratoires avec un volume expiré supérieur au volume inspiré, pause respiratoire en expiration, puis nouvelle phase de respiration régulière.

La neurogénèse ventilatoire appréciée par $V_{T} / T I$ et $P 0.5$ est d'autant plus déprimée que le débit d'alfatésine augmente (Figure 6). Il existe une corrélation significative entre le débit d'alfatésine et la diminution de VT/Tr $(r=-0.420 p$ $<0.01$ ) ainsi qu'entre débit d'alfatésine et $P 0.5$ $(r=-0.425 p<0.01)$. D'autre part il existe une corrélation significative entre VT/Ti et P0.5 $(r=$ 


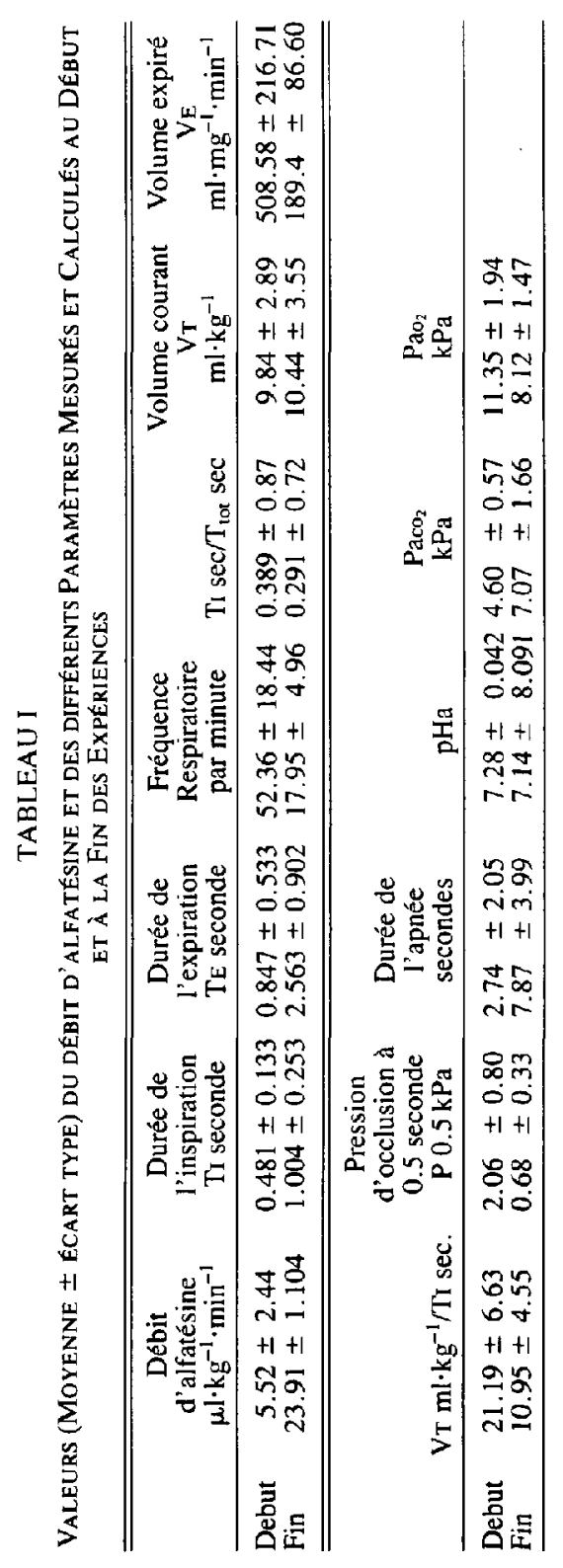




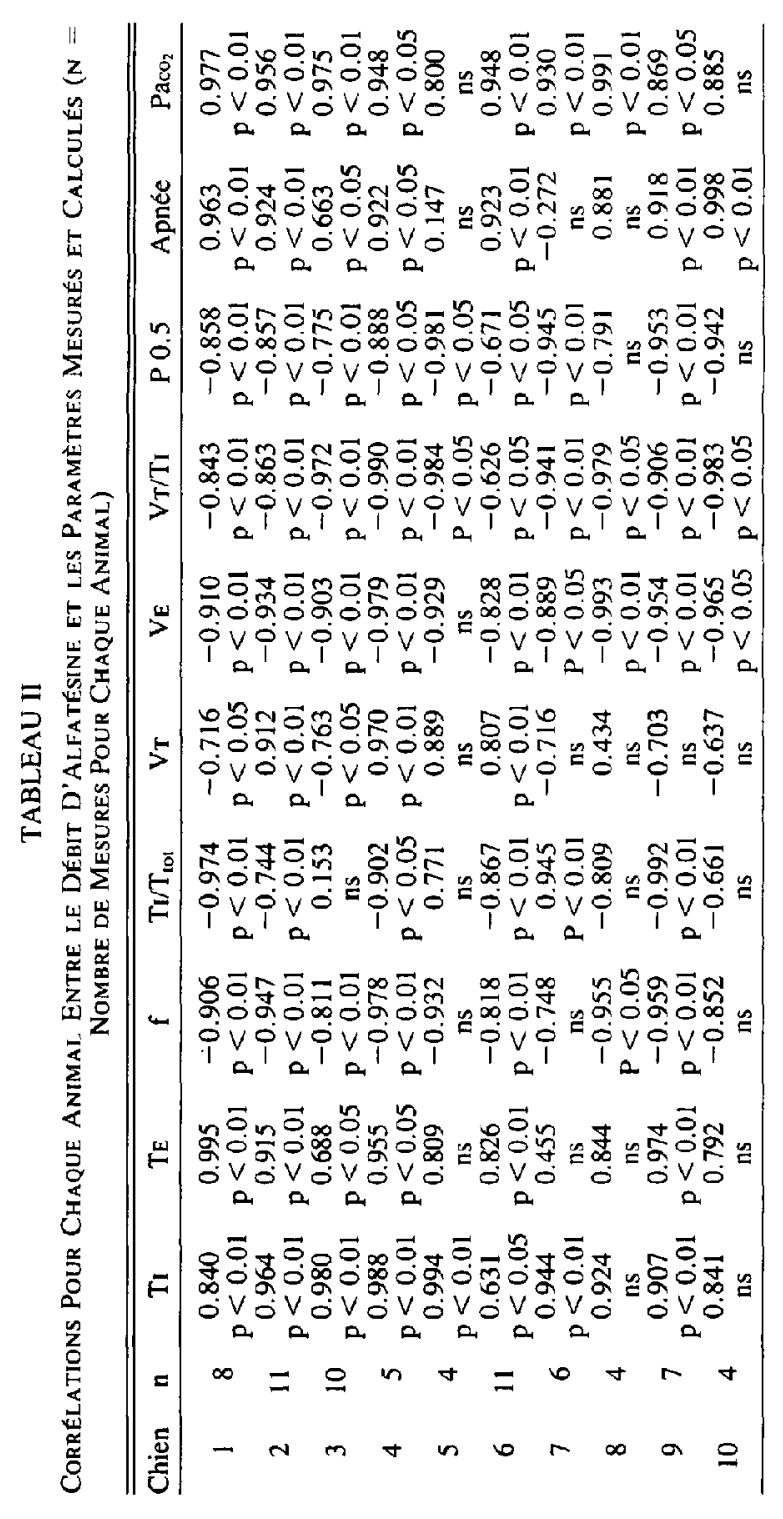



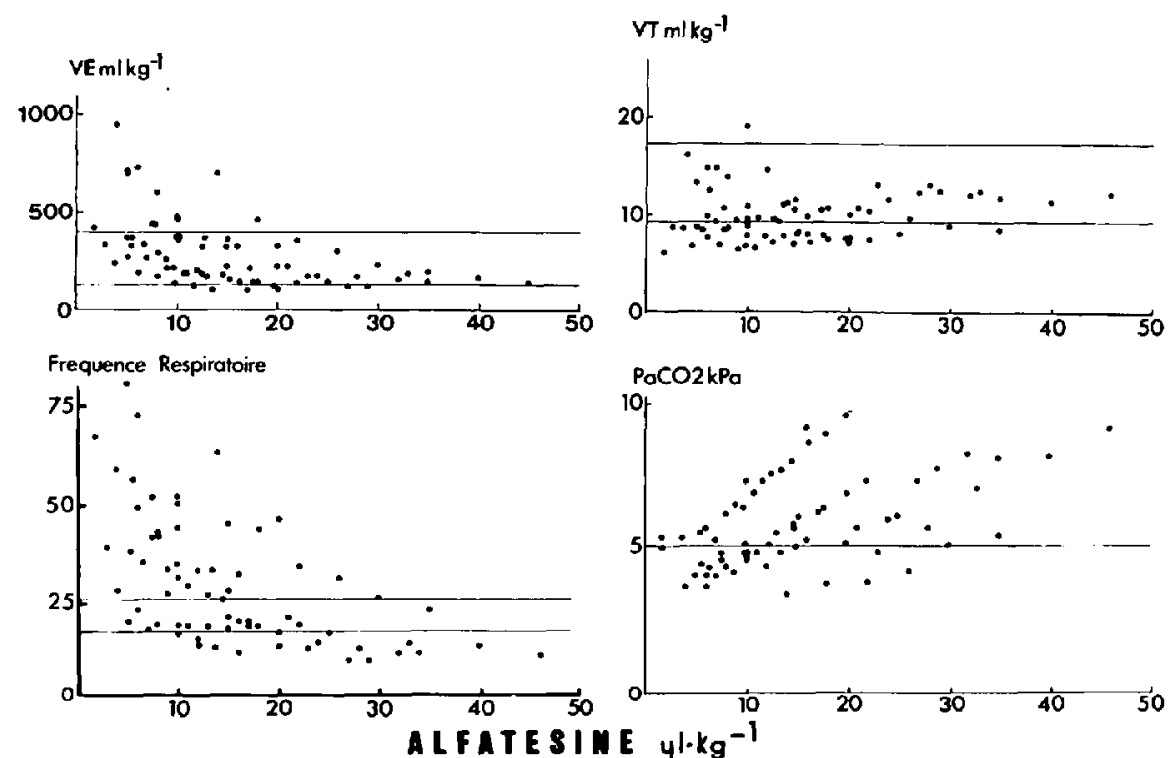

FIGURE 4 Variation du volume expiré par minute $\left(V_{E}\right.$ en $\left.\mathrm{ml} \cdot \mathrm{kg}^{-1} \cdot \mathrm{min}^{-1}\right)$ de la fréquuence respiratoire par minute, du volume courant $\left(\mathrm{ml} \cdot \mathrm{kg}^{-1}\right)$ et de la Pacon $(\mathrm{kPa})$ en fonction du débil d'alfatésine $\left(\mu \mathrm{l} \cdot \mathrm{kg}^{-1} \cdot \mathrm{min}^{-1}\right)$. Les lignes horizontales correspondent aux valeurs normales chez le chien éveillé rapportées par STAHL pour VE. $\mathrm{VT}$. $\mathrm{f}$ et par Green pour Paco.

TABLEAU III

Corrélation Générale ( $N=70$ ) Entre le Débit D'Alfatésine et les Paramètres Mesurés et Calculés

\begin{tabular}{cccccccccc}
\hline \hline $\mathrm{TI}_{\mathrm{I}}$ & $\mathrm{TE}$ & $\mathrm{f}$ & $\mathrm{TI} / \mathrm{T}_{\text {tot }}$ & $\mathrm{VT}$ & $\mathrm{VE}$ & $\mathrm{VT} / \mathrm{TI}$ & $\mathrm{P} 0.5$ & Apnée & Paco $_{2}$ \\
\hline 0.578 & 0.627 & -0.550 & -0.388 & 0.148 & -0.471 & -0.420 & -0.425 & 0.380 & 0.490 \\
$\mathrm{p}<0.01$ & $\mathrm{p}<0.01$ & $\mathrm{p}<0.01$ & $\mathrm{p}<0.01$ & ns & $\mathrm{p}<0.01$ & $\mathrm{p}<0.01$ & $\mathrm{p}<0.01$ & $\mathrm{p}<0.01$ & $\mathrm{p}<0.01$ \\
\hline
\end{tabular}

$0.780 p<0.01)$ pour les différents niveaux d'anesthésie.

Au fur et à mesure que le débit d'alfatésine augmente la durée de l'apnée après occlusion des voies aériennes en cours d'inspiration augmente (Figure 6). Il existe une corrélation significative entre ces deux paramètres pour sept animaux sur 10 et pour l'ensemble des animaux $(r=0.380$ $\mathrm{p}<0.01$ )

\section{Méthodologie}

\section{Discussion}

Des accidents ont été rapportés lors de l'utilisation d'alfatésine chez le chien, ${ }^{13}$ accidents imputés au Crémohor EL. Cela est peut être vrai lors de l'administration intraveineuse directe trop rapide de quantités importantes d'anes- thésique susceptibles d'entrainer des modifications cardiovasculaires importantes. Les réactions anaphylactoïdes (rash, odème, hypotension artérielle systémique) existent également lors de l'induction de l'anesthésie quand elle est réalisée au moyen de la perfusion lente d'une solution diluée de l'anesthésique; mais elles sont bien tolérées. L'hypotension artérielle, constante, est facilement contrôlée par l'administration d'une quantité relativement peu importante d'un soluté de remplissage vasculaire. Cependant, elle explique peut être en partie l'acidose métabolique constatée après l'induction. Certains auteurs ${ }^{14}$ ont déja utilisé sans inconvénient l'alfatésine chez le chien.

L'alfatésine est un anesthésique intéressant quand on désire obtenir une anesthésie intravei- 


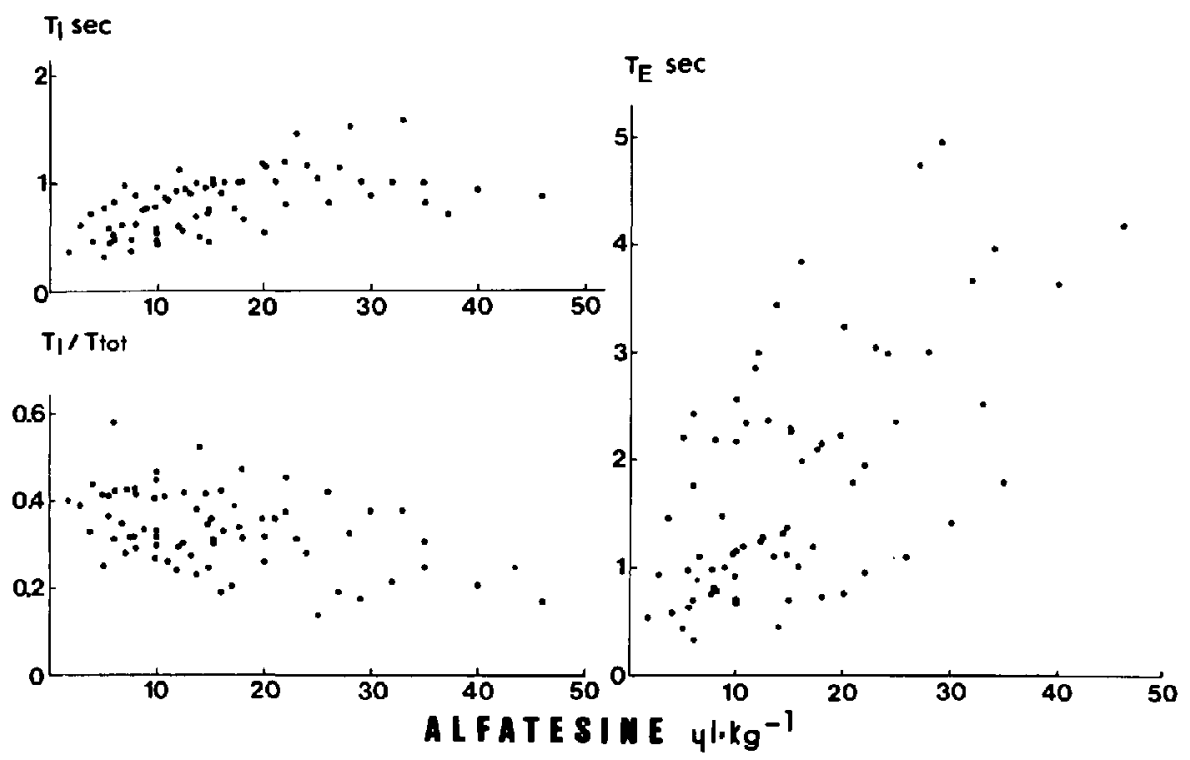

Figure 5 Variation de la durée de l'inspiration ( $\mathrm{T}_{1}$ en secondes), de la durée de l'expiration (TE en secondes) et du rapport de la durée de l'inspiration sur la période $(\mathrm{T} / \mathrm{T} / \mathrm{T}$ ) en fonction du débit d'alfatésine $\left(\mu \mathrm{k} \cdot \mathrm{kg}^{-1} \cdot \mathrm{min}^{-1}\right)$.

neuse stable, en raison de sa pharmacocinétique ${ }^{10}$ quand elle est utilisée en perfusion. Il est possible, si la pharmacocinétique de l'alfatésine est la même chez le chien que chez l'homme, qu'un taux plasmatique stable n'ait pas été réalisé pour chaque palier dans la présente étude, chaque palier n'étant séparé du suivant que par environ trente minutes. Mais, même dans ces conditions, il est peu probable que le taux plasmatique d'alfatésine ait varié pendant les quelques minutes nécessaires aux mesures. L'avantage de l'alfatésine par rapport aux anesthésiques par inhalation est que la concentration sanguine de l'anesthésique est indépendante des modifications de la ventilation, ce qui est important quand on s'intéresse aux effets ventilatoires d'un médicament.

Les animaux ont été laissés en ventilation spontanée à l'air ambiant. Dans ces conditions il est évident que toute dépression ventilatoire entrainant une hypoventilation alvéolaire provoque une hypoxie et une hypercapnie qui à leur tour retentissent sur la ventilation, ce qui rend impossible l'appréciation exacte des effets ventilatoires isolés d'un anesthésique. S'il n'est pas possible de corriger l'hypercapnie, il est par contre possible de corriger facilement l'hypoxémie par l'administration de concentrations adaptées d'oxygène. Mais l'oxygène à lui seul est capable de modifier la ventilation. La dépression ventilatoire qu'il entraine existe déja chez le sujet éveillé, même si elle est discrète et de brève durée. ${ }^{15}$ Elle peut être très importante en cas de dépression du système nerveux central et d'by. poxémie. ${ }^{16,17}$

La neurogénèse ventilatoire peut être appréciée par la mesure de la pression d'occlusion des voies aériennes ${ }^{18}$ et par celle du débit inspiratoire moyen. ${ }^{19}$ La pression d'occlusion est une mesure de l'activité globale des centres respiratoires; elle est indépendante de la résistance et de la compliance du système respiratoire. Il a été démontré lors de tests de stimulation ventilatoire par l'anhydride carbonique que chez le sujet anesthésié seule l'amplitude de l'onde de pression varie alors que la forme ne change pas; il est indifférent de mesurer la pression à n'importe quel temps de l'occlusion. ${ }^{20}$ Dans la présente étude, la pression d'occlusion a été mesurée après 500 millisecondes. Le rapport VT/Ti est le débit inspiratoire moyen. Il est l'expression de l'activité inspiratoire mesurée au niveau des voies aériennes supérieures. On peut considérer ce terme comme la transformation mécanique de la neurogénèse ventilatoire. ${ }^{21}$ Sa diminution peut être due à la 


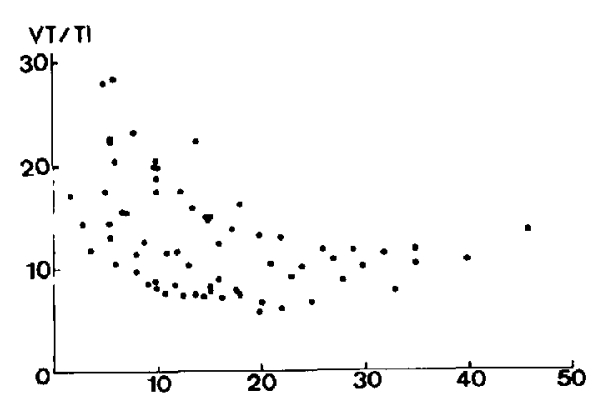

PO. 0 kPo

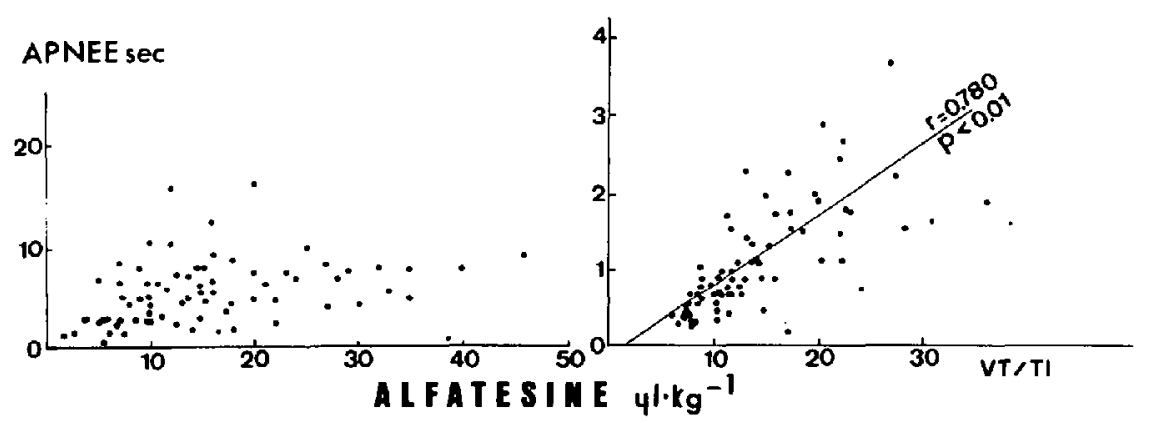

FIGURE 6 Variation du débit inspiratoire moyen (VT ml $/ \mathrm{kg}^{-1} / \mathrm{T}$ I secondes), de la pression d'occlusion trachéale à 0.5 secondes ( $\mathrm{P} 0.5 \mathrm{kPa}$ ) et de la durée d'apnée après occlusion des voies aériennes en cours ou en fin d'inspiration (en secondes) en fonction du débit d'alfatésine $\left(\mu \mathrm{l} \cdot \mathrm{kg}^{-1} \cdot \mathrm{min}^{-1}\right)$. En bas et à droite relation entre Vt/Tı et $P 0.5$ pour l'ensemble des animaux et pour les différents débits d'alfatésine.

diminution de la neurogénèse ventilatoire centrale, à une inadaptation neuro-musculaire, ou bien à l'augmentation de l'impédance respiratoire (augmentation de la résistance ou diminution de la compliance). En cas de modification de $\mathrm{VT} / \mathrm{TI}$ il est possible de faire la part de ce qui revient à une atteinte de la mécanique respiratoire et de ce qui revient à la commande ventilatoire en comparant les variations de $V_{T / T I}$ à celles de la pression d'occlusion. ${ }^{22}$

Durée de L'APNée APrès OCCLUSION DES VOIES AERIENNES EN COURS D'INSPIRATION

L'apnée provoquée par l'occlusion des voies aériennes en cours d'inspiration dépend du refflexe de Beurer Hering. Ce réflexe est puissant chez le chien anesthésie. ${ }^{23}$ Il est modifié par le niveau d'anesthésie. ${ }^{23,24}$ Comme la durée de l'apnée pour un niveau donné d'anesthésie dépend du volume inspiré, ${ }^{24}$ afin d'étudier les variations de la durée de l'apnée en fonction de celles de la profondeur de l'anesthésie, la durée de l'apnée a été rapportée à un volume inspiré arbitraire de $100 \mathrm{~mL}$ (en supposant que la durée de l'apnée, quelque soit le niveau de l'anesthésie, est reliée de manière linéaire au volume inspiré).

\section{RÉSULTATS}

Les résultats de cette étude montrent que l'action de l'alfatésine dépend du débit de l'agent anesthésique (et de la concentration plasmatique qui en découle), les effets étant totalement différents selon le débit. Pour de faibles débits d'alfatésine, correspondant à un stade très léger de l'anesthésie, la ventilation est stimulée comme le prouvent l'augmentation de VE, les valeurs basses de $\mathrm{Paco}_{2}$, les valeurs élevées de $V_{T} / T r$ et de $P 0.5$. Au fur et à mesure que le débit d'alfatésine augmente la ventilation est de plus en plus déprimée.

La stimulation initiale de la ventilation est à rapprocher du tableau décrit chez l'homme par Savege, et al. ${ }^{4} \mathrm{~L}$ 'augmentation de la ventilation est due essentiellement à la tachypnée puisque le volume courant est peu modifié. Pour expliquer 
cette tachypnée on peut invoquer plusieurs mécanismes. Il est probable que l'acidose métabolique constatée chez 8 animaux soit en partie responsable de la stimulation de la ventilation. Gordh $^{25}$ a suggéré que la tachypnée due à l'alfatésine pourrait résulter d'une stimulation des chémorécepteurs artériels périphériques par le solvant de l'alfatésine, le Crémophor EL, qui est également le solvant du propanidide. En effet, le propanidide utilisé dans les mêmes conditions que l'alfatésine, provoque de la même manière une tachypnée avec hyperventilation. Cependant, un effet identique est retrouvé avec d'autres agents anesthésiques comme le penthiobarbital et le méthohexitone qui ne contiennent pas de Crémophor EL. ${ }^{4}$ La stimulation de récepteurs pulmonaires est possible mais peu probable. Cette hypothèse a été avancée par Whitteridge et Bulbring ${ }^{26}$ pour les anesthésiques par inhalation. Mais la persistance de la tachyphnée chez l'animal et chez l'homme après blocage des nerfs vagues ${ }^{27,28}$ n'est pas en faveur d'une telle hypothèse. Plusieurs auteurs ont suggéré qu'une stimulation directe des centres respiratoires pourrait être en cause. ${ }^{29,30}$ L'analyse des effets de faibles débits d'alfatésine sur le régime respiratoire va dans ce sens (Figure 7). Dans ces conditions, l'augmentation de la fréquence, associée à une augmentation de l'activité inspiratoire, est à rapprocher des effets ventilatoires obtenus à la suite d'une stimulation de la formation réticulée. ${ }^{31}$ L'étude réalisée par Mori et $a l^{32}$ met en évidence au cours du stade I de l'anesthésie décrit par ces auteurs une activité réticulaire égale ou supérieure à celle de l'éveil. L'excitabilité du système nerveux central au stade I décrit par Mori et al peut s'accompagner d'une excitation motrice, constatée dans la présente étude. Ces arguments plaident en faveur d'une action centrale de l'alfatésine.

Les effets ventilatoires de l'approfondissement de l'anesthésie constatés dans la présente étude semblent pouvoir être rapportés à une action centrale de l'agent anesthésique. En effets, ces effets ne semblent pas être imputables à des modifications de la mécanique respiratoire en raison de la bonne corrélation qui existe une fois l'anesthésie installée entre la diminution de VT/TI et celles de P 0.5 et du fait que la droite de régression passe pratiquement par l'origine (Figure 6). Ces résultats sont à rapprocher de ceux observés par Nishino et Honda ${ }^{33}$ avec l'halothane chez le chat (mais sur un modèle très différent de celui utilisé dans la présente étude) et du continuum des stades de l'anesthésie décrit

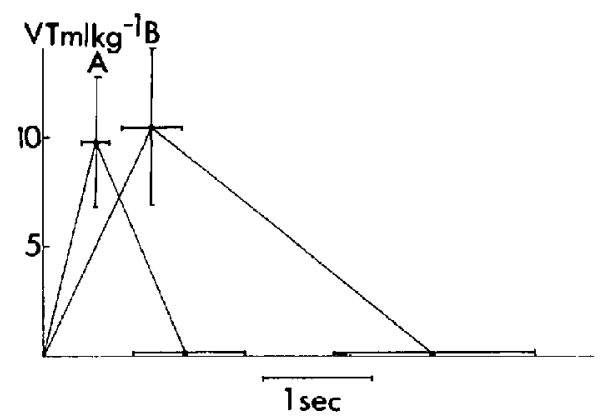

Figure 7 Valeurs du volume courant $\left(V_{T} \mathrm{ml} \cdot \mathrm{kg}\right.$ \pm écart type), de la durée de l'inspiration (Ti secondes \pm écart type), de la durée de l'expiration (Te secondes \pm écart type), au début (A) et en fin (B) d'expérience.

par Winters et Wallach. ${ }^{34}$ Chez le chat vagotomisé et ventilé artificiellement. Nishino et Honda constatent une augmentation de la fréquence des décharges phréniques en cas d'anesthésie légère avec l'halothane, puis une diminution de ces décharges quand on approfondit l'anesthésie. Ces modifications de l'activité respiratoire en fonction de la profondeur de l'anesthésie correspondent au schéma proposé par Winters et Wallach pour l'halothane, c'est à dire stimulation du système nerveux central en cas d'anesthésie légère, puis dépression du système nerveux central. Si on se base uniquement sur les effets ventilatoires de l'alfatésine, constatés dans la présente étude, l'alfatésine ferait partie de la catégorie d'agents anesthésiques, tel l'halothane, qui agissent de façon biphasique sur le système nerveux central, stimulation puis dépression, en fonction de la profondeur de l'anesthésie, la dépression ventilatoire survenant malgré l'hypoxie et l'hypercapnie croissantes. II est toutefois possible, en raison des différences qui existent entre les espèces, que ces observations ne puissent être extrapolées à l'homme. De plus, dans l'état actuel des techniques dont on dispose, les mécanismes de cette action centrale ne peuvent être élucidés que par des études neurophysiologiques chez l'animal, et pour les expliquer force est de se borner à des hypothèses reposant sur des études réalisées sur des modèles très différents de celui de l'animal entier. Ainsi, pour expliquer la tachypnée de l'anesthésie superficielle, on peut imaginer que des modifications fonctionnelles du tronc cérébral provoquées par l'alfatésine pourraient être responsables d'un accroissement du recrutement des décharges inspiratoires, sans altérer le seuil de déclenchement de l'"off switch", mais avec 
réduction de sa durée d'activité. Dans ces conditions, les contrôles corticaux qui normalement réduisent graduellement les effets d'un Éveil sur les décharges phréniques ${ }^{31}$ seraient inefficaces. Au cours de l'approfondissement de l'anesthésie, il est possible que la conservation du volume courant soit due à l'hypoxémie et à l'hypercapnie qui se développent progressivement. Le seuil de déclenchement de 1" off switch" resterait inchangé bien que l'hypercapnie renforce l'influence inhibitrice dont il est l'objet. ${ }^{35}$ Dans ces conditions, la prolongation de TI ferait suite à une diminution de l'activité inspiratoire. L'activation du centre générateur inspiratoire par l'hypercapnie ${ }^{35}$ serait insuffisante par rapport aux effets dépresseurs de l'anesthésie. L'importante prolongation de TI pourrait également être en rapport avec la baisse d'activité de l'oscillateur réticulaire provoquée par ce type de drogue. ${ }^{36}$ L'hypoxie qui prolonge considérablement la durée de l'apneuse chez le chat dont les centres pneumotaxiques ont été détruits et les vagues sectionnées ${ }^{37}$ pourrait également agir sur l'oscillateur réticulaire dans le même sens que certains anesthésiques. ${ }^{37}$ Par contre la réponse des chémorécepteurs périphériques à l'acidose respiratoire et à l'hypoxie devrait stimuler les centres respiratoires si ces derniers étaient en mesure de répondre. Mais en cours de l'anesthésie, la réponse à l'hypercapnie est diminuée, ainsi que la réponse à l'hypoxie, au moins pour certains agents anesthésiques. ${ }^{38-40}$ De même que Tı, TE semble peu influencé par l'acidose respiratoire et TE augmente quand on approfondit l'anesthésie. Cette prolongation pourrait être due à une modification du rapport des activités dans le centre respiratoire entre les elements inhibiteurs et les elements facilitateur, dans la mesure ou il est admis que certains neurones du "switching system" déchargent en permanence au cours de l'expiration. ${ }^{41,42} \mathrm{~L}$ 'accroissement de l'activité vagale, corrélée avec l'approfondissement de l'anesthésie, déja constatée par d'autres auteurs, pourrait également être liée à la tendance à la prolongation de l'activité du "switching system" au cours de l'expiration.

Corrélation des Modifications VentilaTOIRES OBSERVEES AVEC LE DÉBIT D'ALFATESINE: ESSAI D'APPRÉcIATION DE LA PROFONDEUR DE L'ANESTHÉSIE

L'appréciation de la profondeur d'anesthésie est importante en recherche expérimentale animale puisque les effets observés peuvent varier selon la profondeur de l'anesthésie. L'idéal serait de pouvoir déterminer les différents niveaux d'anesthésie chez le même animal, mais aussi de les comparer entre différents animaux. Dans l'état actuel des connaissances le problème n'est pas résolu. ${ }^{43}$ Même l'utilisation du MAC (concentration alvéolaire minimale d'un agent anesthésique par inhalation) n'est pas exempte de critiques. ${ }^{44} \mathrm{C}$ 'est la raison pour laquelle une corrélation a été recherchée entre le débit d'alfatésine et les paramètres ventilatoires mesurés et calculés afin de savoir si il était possible à partir de l'un ou de plusieurs de ces paramètres d'apprécier la profondeur de l'anesthésie.

Il est probable en raison de la pharmacocinétique de l'alfatésine qu'au cours des paliers successifs d'une durée de trente minutes chacun, la concentration plasmatique de l'anesthésique et par conséquent sa concentration au niveau du système nerveux central, aient été de plus en plus grandes. La dépression ventilatoire croissante qui suit la stimulation initiale va dans ce sens. Si pour chaque animal il existe une bonne corrélation significative entre le débit d'alfatésine et certains paramètres (fréquence respiratoire, volume expiré par minute, $\mathrm{Paco}_{2}, \mathrm{VT} / \mathrm{TI}$ ), les corrélations générales pour l'ensemble des animaux, même si elles sont significatives, ne sont pas étroites. D'autre part, les valeurs observées pour un débit donné d'alfatésine, sont très variables d'un animal à l'autre. Enfin l'observation visuelle des variations de $V_{E}, f, V_{T} / T I$ et $P$ 0.5 montre que la relation entre ces paramètres et le débit d'alfatésine n'est pas linéaire et qu'elle revêt plutôt l'allure d'une courbe exponentielle. En conséquence, si chez un animal donné certains paramètres ventilatoires permettent de suivre l'approfondissement de l'anesthésie, aucun de ces paramètres ne permet de comparer différents niveau d'anesthésie d'un animal à l'autre.

\section{BIBLIOGRAPHIE}

1. Miller, A.H. Ascending respiratory paralysis under general anesthesia. J.A.M.A. 84: 201-202 (1925).

2. GUEDEL, A.E. Inhalation anesthesia 2nd ed. New York The Mac Millan Co pp 10-52 (1951).

3. ChILd, K.J., Davis, B., Dodds, M.G. \& TwisSEL, D.J. Anaesthesic, cardiovascular and respiratory effects of a new steroidal agent CT 1341: a comparison with other intravenous anaesthetic drugs in the unrestrained cat. Brit. J. Pharmacol. 46: 186-200 (1972)

4. Savege, T.M., Ross, L., Foley, E.I. \& MaXWELL, M.P. A comparison of the cardiorespiratory effects during induction of anaesthesia of 
althesin with thiopentone and methohexitone. Post. Med. J. 48. Suppl. 2: 66-72 (1972).

5. Tombin, P.J. The respiratory effects of althesin. Post. Med. J. 48 Suppl. 2: 85-89 (1972).

6. Bouyard, P., Cotte, G., Mesdjian, E., GasTAUT, J.A. , JaDOT, G. \& MarTelli, M. Place du CT 1341 (Alfatésine) parmi les stéroïdes anesthésiques: ses propriétés pharmacodynamiques. Ann. Anesth. Franç. 14: 481-490 (1973).

7. Du CaILAR, J. Less effets respiratoires du CT 1341. Ann. Anesth. Franç. 14: 535-539 (1973).

8. HaLl, G.M., Whitwam, J.G. \& Morgan, M. Some respiratory effects of althesin. Brit. J. Anaesth. 45: 629-632 (1973).

9. Fantera, A. \& MaURINa, M. Impiego dell'althesin nell'induzione endovenosa dell'anestesia generale, e valutazione dei suoi effetti cardiocirculatori e respiratori. Acta Anaesth. Ital, 27: 241-256 (1976)

10. Sear, J.W. \& Prys-Roberts, C. Alphadione and minaxolone pharmacokinetics. Ann. Anesth. Franç 22: 142-148 (1981).

11. GREEN, C.J. Animal anaesthesia Ist ed. London Laboratory Animals LTD pp 207 (1979).

12. STAHL, W.R. Scaling of respiratory variables in mammals. J. Appl. Physiol. 22: 453-460 (1967).

13. Child, K.J., Currie, J.P., Davis, B., Dodos, M.G., Pearce, D.R. \& Twissell, D.J. The pharmacological properties in animals of CT 1341. A new steroid anaesthetic agent. Brit. J, Anaesth. 43: 2-13 (1971)

14. Michalot, G., Girardet, P., Grimbert, F., Piasentin, D. Stieglitz, P. 24 hour althesin fentanyl angesthesia in dogs. Time course of haemodynamic changes. Br. J. Anaesth. 52: 19-22 (1980).

15. Dejours, $P$, Chemoreflexes in breathing. Physiol. Rev. 42: 335-358 (1962).

16. Mosso, A. L'apnée produite par l'oxygène. Arch. Ital. de Biol. 34: 138-157 (1904).

17. Marshall, E.K. \& Rosenfeld, M. Depression of respiration by oxygen. J. Pharmacol. Exptl. Therap. 57: 437-457 (1936).

18. Milic-Emili, J., Whitelaw, W.A. \& DeRENNE, J.PH. Occlusion pressurc simple measurement of the respiratory centers output. $\mathrm{N}$. Engl. J. Med. 293: 1029-1030 (1975)

19. DeRenNe, J.PH. Méthode d'investigation elinique des mécanismes régulateurs de la ventilation Bulletin Européen de physiologie respiratoire 13: 681-727 (1977)

20. Derenne, J.Ph,, Couture, J., Iscoe, S., Whitelaw, W.A. \& Milic-EmiLi, J. Occlusion pressure in man breathing $\mathrm{CO} 2$ under methoxyflurane anesthesia. J. Appl. Physiol. 40: 805814 (1975).

21. Gautier, H. Effets compares de stimulations respiratoires spécifiques et de l'activité mentale sur la forme du spirogramme de l'homme. Journal Physiologie (Paris) 61: 31-44 (1969).

22. MiLic-EMiLI, J. Recent advances in the evaluation of respiratory drive. Int. Anesthesiol. Clin. 15: 39-58 (1977)

23. WIDDICOMBE, J.G. Respiratory reflexes in man and other mammalian species. Clin. Sci. 21: $163-170$ (1961).
24. Bouverot, P., Crance, J.P. \& Dejours, P. Factors influencing the intensity of the BreuerHering inspiration inhibiting reflexe. Respir. Physiol. 8: 376-384 (1970).

25. GORDH, H. The respiratory effects of althesin. Discussion. Postgrad. Med. J. 48, Suppl. 2: 89 (1972).

26. Whitteridge, D. \& Bulbring, E. Changes in the activity of pulmonary receptors in anesthesia and their influence on the respiratory behaviour. J. Pharmacol. Exp. Therap. 81: 340-359 (1944).

27. Guz, A., Noble, M.I.M., TRenchard, D., Cochrane, H.L. \& MaKeY, A.R. Studies in the vagus nerves in man: their role in respiratory and circulatory control. Clin. Sci. 27: 293-304 (1964).

28. Mazzarelli, M., Haberer, J.P., Jaspar, N. \& MiserRoChI, G. Mechanism of halothane induced tachypnea in cats. Anesthesiology 51: 522-527 (1979)

29. NGaI, H.S., KatZ, R.L. \& FaHRIE, S.E. Respiratory effects of tricholorethylene, halothane and methoxyflurane in the cat. J. Pharmacol. Exp. Ther. 148: 123-130 (1965).

30. Paskins, S., Skowsted, P. \& Smith, T.C. Failure of the Hering-Breuer reflex to account for tachypnea in anesthetized man. Anesthesiology 29: 550-558 (1968).

31. Hugelin, A. \& Cohen, M.I. The reticular activating system and respiratory regulation in the cat. Ann. NY. Acad. Sci. 109: 586-603 (1963)

32. Mori, K., Winters, W.D. \& Spooner, C.E. Comparison of reticular and cochlear multiple unit activity with auditory evoked response during various stages induced by anesthetic agents II. EEG Clin. Neuro. Physiol. 24: 242-248 (1968).

33. Nishino, T. \& Honda, Y. Changes in the respiratory pattern induced by halothane in the cat. Br. J. Anaesth. 52: 1191-1197 (1980).

34. Winters, W.D. \& Wallach, M.B. Drug induced states of CNS excitation: a theory of hallucinosis. In: Psychomimetic drugs (Efron.D.H. ed.) 193-214 Raven Press New York (1970)

35. von Euler, C. \& TRIPPEnBaCh, T. Excitability changes of the inspiratory "off-switch" mechanism tested by electrical stimulation in nucleus parabrachialis in the cat. Acta Physiol. Scand. 97: 175-188 (1976).

36. Caille, D., Vibert, J.F. \& Bertrand, F. Pentobarbitone effects on respiration related units: selective depression of bulbo-pontine reticular neurons. Resp. Physiol. 35: 201-216 (1979).

37. Hugelin, A. Does the respiratory rhythm originate from a reticular oscillator in the waking state? In: The reticular formation revisited (Hobson, J.A. and Brazier, M.A.B. ed.) Raven Press New York pp 261-274 (1980).

38. WEISKOPF, R.B., RAYMOND, L.W. \& SEVERINGHAUS, J.W. Effects of halothane on canine respiratory responses to hypoxia with and without hypercarbia. Anesthesiology 4I: 350-360 (1974).

39. Duffin, J., Triscott, A. \& Whitwan, J.G The effect of halothane and thiopentone on ventilatory responses mediated by the peripheral 
chemoreceptors in man. Br. J. Anaesth. 48: 975-981 (1976)

40. Knill, R.L., Manininen, P.H. \& Clement, J.L. Ventilation and chemoreflexes during enflurane sedation and anaesthesia in man. Canad. Anaesth. Soc. J. 26: 353-360 (1979).

41. KNox, C.K. Characteristics of inflation and deflation reflexes during expiration in the cat. $\mathrm{J}$. Neurophysiol. 36: 284-295 (1973).

42. CoHEN, M.I. Switching of the respiratory phases and evoked phrenic responses produced by rostral pontine electrical stimulation. J. Physiol. (London) 217: 133-158 (1971).

43. Artusio, J.F., Corssen, G., Dornette. W.H.L. \& REVES, J.G. Monitoring depth of anesthesia. In Clinical Anesthesia: Monitoring in anesthesia. W.H.L. Dornette Ed., F.A. Davis Company Philadelphia pp 212-222 (1973).

44. WAUD, B.E. \& WAUD, D.R. On dose-response curves and anesthetics (Editorial). Anesthesiology 33: $1-4$ (1970).

\section{ABSTRACT}

In 10 dogs the authors studied the effects of increasing doses of alfathesin on ventilation ( $\dot{V}_{E}$, frequency, $V_{T}$, blood gases), on the ventilatory pattern ( $T I, T E, T I / T_{10 t}$ ), on the neurological initiation of ventilation (VT/Ti, occlusion pressure at 0.5 seconds), and on the Hering-Beurer reflex (duration of apnoea after occlusion of the airway at the end of inspiration). The results were compared with normal values taken from the literature. The correlation between the dose of alfathesin and the measured or calculated parameters was examined.

Ventilation was stimulated by low doses of alfathesin, a stimulation characterized by tachypnoca without change in tidal volume. Deepening of anaesthesia was accompanied by progressively increasing depression of respiration (diminution of $\dot{V}_{E}$, of frequency, of $V_{T} / T_{I}$ and increase of $\mathrm{PaCO}_{2}$ and of the duration of apnoea).

The mechanisms of the initial stimulation of ventilation and of respiratory depression are discussed.

The authors conclude that the action of alfathesin on the central nervous system is biphasic, with stimulation during light anaesthesia followed by depression with associated depression of ventilation, despite increasing hypoxia and hypercapnia. 